\title{
Motion Studies: an art and science collaboration
}

Fernanda D'Agostino 5711 SW Boundary Street

Portland, Oregon 97221

nandada@aol.com www.fernandadagostino.com
Harry Dawson

harry@harrydawson.com www.harrydawson.com
Bret W. Tobalske

Ornithology and Biomechanics

Field Research Station at Fort Missoula,

Division of Biological Sciences,

University of Montana,

Missoula, Montana, 59812

bret.tobalske@mso.umt.edu

Motion Studies brings together investigations at the intersection of Art and Science. At the core of the project are motion studies undertaken in Dr. Bret Tobalske's flight laboratory at the University of Portland and translated into video, first at the flight lab and later in Fernanda D'Agostino's studio. Motion Studies uses a fluid dynamics imaging system known as digital particle velocimetry to compare the nature of birds' flights in different conditions. The footage is later edited into an experimental art video that combines location footage, archival imagery and a digitally altered sound track of wild birds' calls. Motion Studies has been exhibited around the world as both a single channel video and as part of a video installation. Insights gained while working on the art video have led to promising new scientific research directions for the team and to a series of related art works.

Art and science. Digital particle velocimetry. Scientific imaging software. Interdisciplinary collaboration. Public art. Installation. Video art. Bird flight.

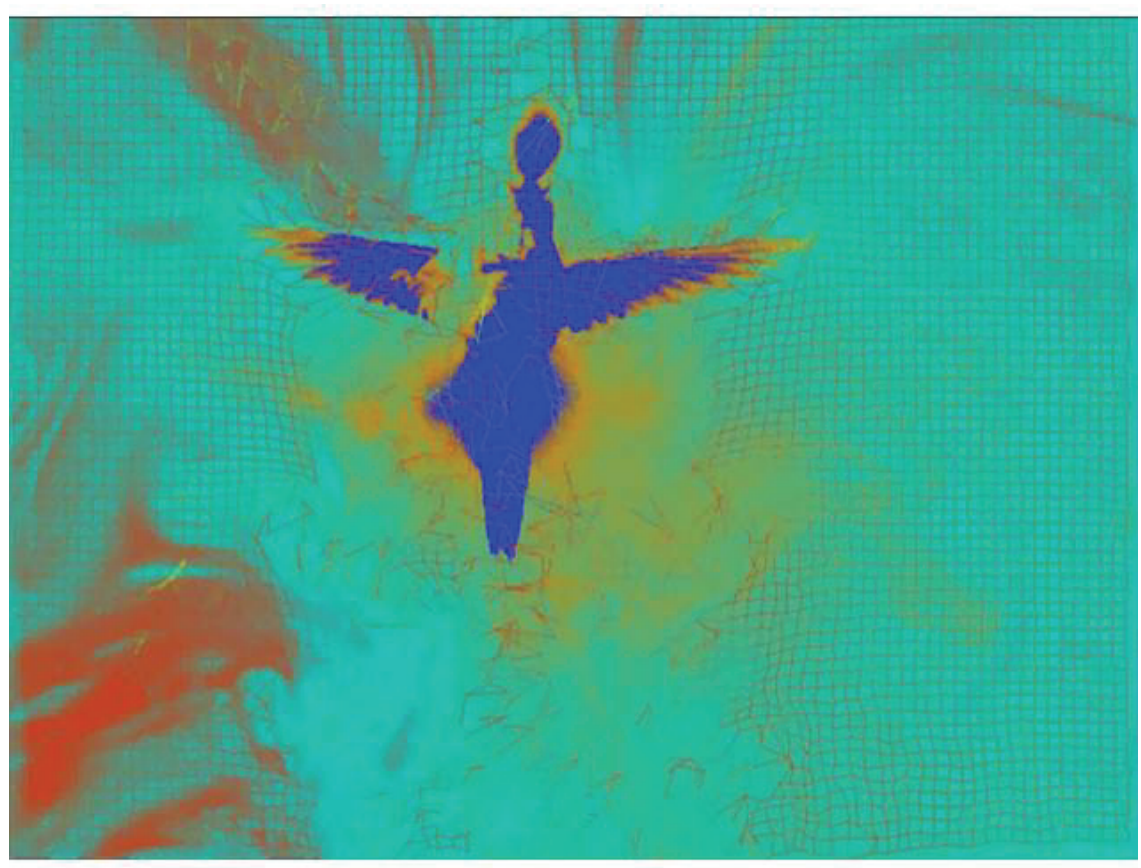

Figure 1: Still from Motion Studies 


\section{INTRODUCTION}

Artists and scientists share the desire to see beneath the surface of things, leading to parallel developments between the two disciplines. Observation and experimentation are at the heart of both fields and because of that artists and scientists are natural allies. Recent developments in specialised digital imaging systems in both fields have created an unprecedented ability to unravel the processes underlying the beauty and mystery of nature. Video installation artist Fernanda D'Agostino and biomechanist Dr. Bret Tobalske have formed an alliance to create work which exposes new developments in our understanding of the physics of flight to a wider audience.
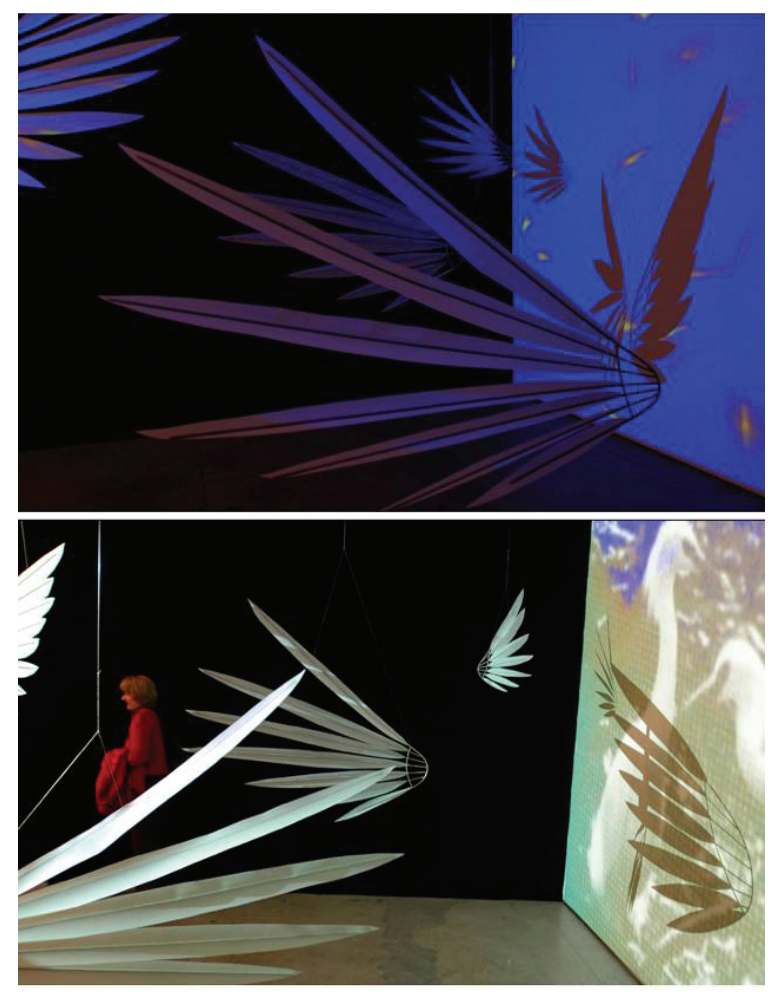

Figures 2 \&3: Scale played an important role in viewers' experience of the Motion Studies installation

Motion Studies investigates the intersection of Art and Science. At the core of the project are ornithological motion studies captured in Dr. Bret Tobalske's wind tunnel at his flight lab. These flight studies are then translated into video at the lab and in D'Agostino's studio. Motion Studies uses a fluid dynamics imaging system known as digital particle velocimetry to examine the structure of the wake of flying birds. The fluid motions of the air currents around the bird are made visible by the application of colours and grids that respond to the flow of air generated by the bird's flight. At times this footage is like a moving abstract painting at other times the bird's flight is more explicit. Combined with these images, is footage of bird mating dances and flights, shot during the migration of cranes along the Columbia River, and of Vaux's Swifts Chaetura vauxi) returning to their roosts during their annual migration. This footage was shot on location with a high speed, high definition camera that renders playback in slow motion. An interesting development in the collaboration came about as a result of experimenting by applying digital particle image velocimetry to footage shot in the wild. When the footage of the flocking swifts was processed we discovered that some of the same descriptions of fluid dynamics may apply to the patterns of movement within the flock as apply to the air currents produced by individual birds. This is an area of inquiry we are eager to pursue in the future. Motion Studies has also been exhibited as a video installation projected on sculptural screens. The footage is projected on a series of stainless steel and hand painted Mylar 'wings' suspended in the air. The wings respond to the slightest air current, creating an analogous experience to Dr. Tobalske's investigations of the role of air currents in birds' flight. The video projections on translucent surfaces create an activated space that viewers can move around and through, giving them a physical experience of being 'within the flock'. In the United States funding for National Science Foundation grants requires that the applicant provide for ways to make their work accessible to a more general public outside the world of academia. Our shared exhibitions, artists' talks and participation in conferences and film festivals around the world give lay viewers a glimpse into the mysteries of flight being unravelled in Dr. Tobalske's flight laboratory. At the same time insights gleaned by the artist into the pervasive role fluid dynamics plays in the organisation of the natural world have led to new artworks for public places and even to engineering innovations in the development of artist designed structures. Because each member of our team brings to the collaboration different skill sets and motivations, a synergy has emerged that has allowed for new developments in both disciplines.

\section{TECHNICAL SPECIFICATIONS OVERVIEW}

The first segment of Motion Studies combines scientific imagery shot and edited in Dr. Bret Tobalske's Ornithology laboratory using a LaVision, GmbHDigital Particle Image Velocimetry system (DaVis 7.1 software), with archival footage of Egrets shot in the Everglades at the turn of the $20^{\text {th }}$ century. The contemporary location footage that comprises the second half of the video program was shot on location at Sauvie Island Wildlife Refuge, outside of Portland, Oregon, for Sandhill Crane Migration and in Portland proper for Vaux Swift Migration. For this field effort, Harry Dawson used a Panasonic HDX900 camera at 1000 frames 
per second at full DVCPROHD1080. Location footage was formatted in Adobe After EffectsCS3 (Adobe Systems, Inc.) to match the pixel aspect ratio, aspect ratio and frame size native to the LaVision system.. The location footage was then analyzed using DaVis. In its scientific application, this LaVision software applies colours and grids to footage of flying birds photographed in rigorously controlled conditions. (See below) This enables researchers to analyze both the bird's movement and the direction and velocity of the surrounding air. Much of the first segment of Motion Studies employs the LaVision software system in precisely this way.

In working with the location footage shot by Dawson, D'Agostino used the colour palettes of the LaVision software in a more intuitive, aesthetically driven way. The artist's work, however, opened up potential new areas of scientific research which will be elaborated on later in this text. Finally location footage, archival footage and flight laboratory footage were all brought together in Adobe Premiere Pro CS3 (Adobe Systems, Inc.) editing software to create the 12 minute 24 second long experimental art video. The LaVision DPIV system only renders 12 second segments of imagery. Continuity was achieved through frame matching in both the LaVision software and in Premiere during editing and post production. A digitally altered sound track of bird calls accompanies the video and underscores the mysterious mood the artist sought to create.

For the installation exhibition of the project, at the Elizabeth Leach Gallery in Portland in April 2008, nine wing forms based on stills from the video were fabricated using stainless steel rods and hand painted velum. The vellum acted as a dual sided projection surface and yielded a viewing angle of 180 degrees. The wings were ceiling mounted in a room 16 ' $\mathrm{H}$ x $16^{\prime} \mathrm{W}$ by $22^{\prime} \mathrm{D}$, using monofilament and swivel mounts at each attachment point, so that each wing rotated independently with the slightest air current. The largest wing spanned seven feet with a four foot depth. As viewers moved about the room the 'architecture' of the space was always in flux as the movement of the wings constantly reshaped the space. Two of the walls of the gallery were draped with light absorbing fabric while the third wall acted as another projection screen. The fourth wall was used for a corollary installation on scientific glass blowing based on the principals of fluid dynamics. As the wings moved in the air, they caught the video projections and created a mesmerising space for the viewer to enter, investing the science of flight with a sense of mystery.

\subsection{Description of Flow Visualisation and Particle Image Velocimetry}

To visualise and measure the flow of air around a flying bird, we seed the air with a fog of sub-micron sized particles of olive oil. Then we use a dualcavity pulsed laser to illuminate the flowfield. The oil particles reflect the $532 \mathrm{~nm}$ (green) laser light. These particles are nearly neutrally buoyant, so they move freely along with the air. A computerbased system (LaVision, $\mathrm{GmbH}$, DaVis 7.1 software) is used to synchronise the laser flashes with pairs of digital video images $(1,376 \times 1,040$ pixel). The technique of synchronising is called 'frame straddling' because one laser flash occurs at the end of the exposure time for the first image in a pair, and the second laser flash occurs at the start of the exposure time for the second image. The time between laser flashes varies from 200-400 microseconds. To calculate particle velocity, we use cross-correlation of the paired images. We employ an adaptive multipass with an initial interrogation area of $64 \times 64$ pixels and final area of $16 \times 16$ pixels with $50 \%$ overlap. Vector fields are post-processed using a median filter (strong removal if difference relative to average more than 2 * r.m.s. of neighbours and iterative reinsertion if less than 3 * r.m.s of neighbours), removal of groups with less than 5 vectors, fill of all empty spaces by interpolation, and one pass of $3 \times 3$ smoothing. The error estimated for the velocity $(\mathrm{m}$ $\mathrm{s}^{-1}$ ) measurements is $5 \% \pm 0.5 \%$ including contributions due to a correlation peak of 0.1 pixels, optical distortion and particle-fluid infidelity. The birds were flown in a variable-speed wind tunnel that features a 6:1 contraction. The birds fly within a clear working section that is $60 \times 60 \mathrm{~cm}$ in cross section. 


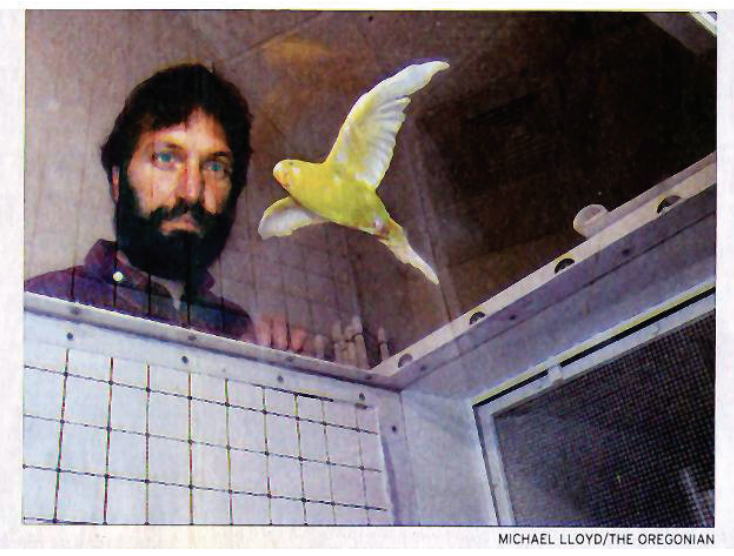

Figure 4: Dr. Tobalske with one of his subjects, a budgerigar (Melopsittacus undulatus) flying at $10 \mathrm{~m} \mathrm{~s}^{-1}$ in the flight chamber of the wind tunnel

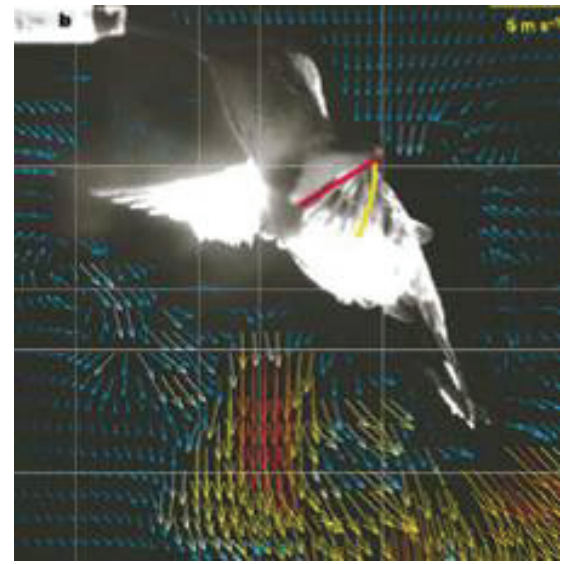

Figure 5: Image of the near wake of a hovering rufous hummingbird (Selasphorus rufus) as it is typically seen in the scientific literature (Warrick et al.2005); Note the application of colours and grids indicating movement and velocity

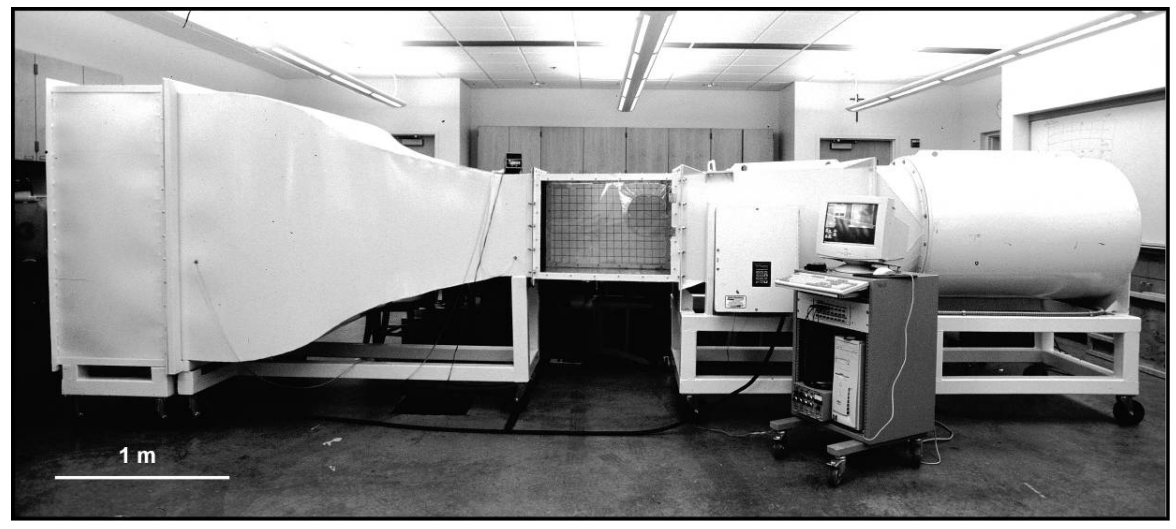

Figure 6: The Wind Tunnel at the Flight Laboratory

\section{ART AND SCIENCE COLLABORATION; DISCOVERIES ACROSS DISCIPLINES AS A RESULT OF MOTION STUDIES}

Although the primary collaborators in Motion Studies come from radically different disciplines, we have in common a deep commitment to intense observation and to using digital imaging to reveal hidden phenomena. Dr Tobalske's observations, and the technology he has created to facilitate them, are aimed at unravelling the mysteries of flight and translating them with mathematical precision.

D'Agostino's observations of birds, and their presence as a motif in her work for over twenty years, is based more on intuition and a sense of wonder that their migrations, flights and rituals can be so beautiful, and yet so utterly foreign to our own experience. Her collaboration with Tobalske began a decade ago when she learned of his work from a magazine and approached him about using flight video in an earlier Installation. (Theatre of Memory, 2001)
When Tobalske began using Digital Particle Velocemetry in his research, he thought an artist could do something with the system because of the way layers of colour and grids were used to visualise previously invisible phenomena. He suggested that D'Agostino learn the program and explore whether it could be used to create experimental video.

In 2005 D'Agostino began spending time in the lab, observing experiments in the wind tunnel, and learning to use the LaVision software that provided the digital imagery and computational analysis critical to Dr, Tobalske's work. An initial five minute long clip was produced using only imagery from the flight lab. Because the ability to duplicate results is critical to the scientific method, the footage coming out of the flight lab was somewhat repetitious.To create a more varied piece and to suggest a sense of mystery, D'Agostino began layering archival footage of wild birds with the more controlled flights from the lab. To expand her range of material D'Agostino brought cinematographer Harry Dawson into the team, in order to capture some of 
the incredible migratory bird flights along the Columbia River.

Dawson's work as a cameraman on major motion pictures, gave him the technical knowledge and skills to capture movement and behaviours in wild birds that are difficult for a casual observer to catch with the naked eye. Using the Panasonic HDX900 camera, a telephoto lens and shooting from a quarter mile distance, Dawson was able to capture mating dances, flocking behaviour, and individual flights of Sand Hill Cranes during their annual migration along the Columbia River. This footage became the wild heart of the Motion Studies installation, providing not only the longest segment of the video, but also the freeze frame images of wing movement that were abstracted into the stainless steel and Mylar wing/screens that animated the installation space viewers moved through.

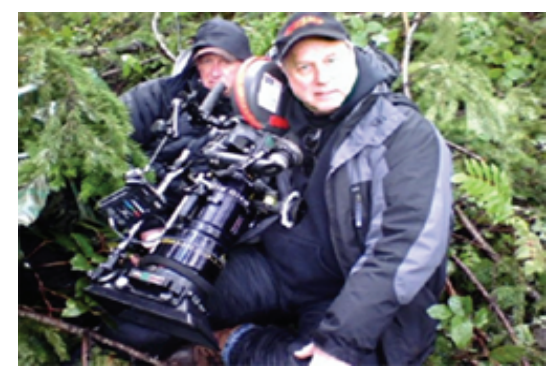

Figure 7: Cinematographer Harry Dawson

Because Dawson filmed at 1000fps and from a distance where the birds' behaviour was not disturbed by our presence, we were able to shoot wild footage with nearly as much control and precision as was achieved in the lab. Viewing the location footage, Tobalske saw that working with a trained cinematographer opened up the possibility of conducting research on the biomechanics of flight based on birds' behaviour in their natural habitat, something he had previously thought impossible due to the difficulty of tracking their flights in an uncontrolled situation. The artistic impulse to expand the range of our subjects inadvertently opened new possibilities for research. Tobalske also observed that the flocks of Vaux's Swifts spiralling around a huge chimney/roost in Portland, seemed to be moving as a vortex, obeying some of the same rules of fluid dynamics operating about the wings of individual birds in flight. Figure 8 illustrates a preliminary attempt at analyzing body motion of birds engaged in flocking behaviour. In the laboratory setting, the LaVision Software assigns velocity to the displacement of micron-sized particles of olive oil in the mist filling the wind tunnel chamber. Moving wireframe grids are generated based on the software's ability to track individual droplets as they move through the air in response to the wind and the wing beats of the bird. In our preliminary flocking experiment Dr. Tobalske treated the individual birds in the flock as particles, thus allowing the software to map the dynamics of the flock's motion around the roost. These preliminary observations opened up new research possibilities for $\mathrm{Dr}$. Tobalske to study the biomechanics of bird flight in natural habitat and to study the physics of flocking behaviour using a large-scale fluid-motion model. Our proposal to move forward with this research was awarded a Lindbergh Foundation Honour Award in 2009 and we are seeking additional funding to continue studying flight in the wild.

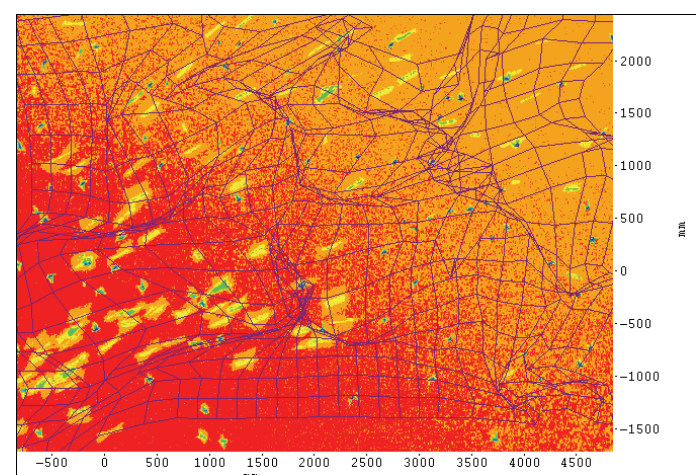

Figure 8: Preliminary experiment analysing location footage to understand the Physics of flocking behaviour

\subsection{Fluid Dynamics and Public art}

Along with new scientific research directions, major art works have been achieved as a result of the collaboration. Celestial Navigations a permanent outdoor video installation at Seattle's SeaTac airport has recently been installed, and includes footage from Motion Studies.

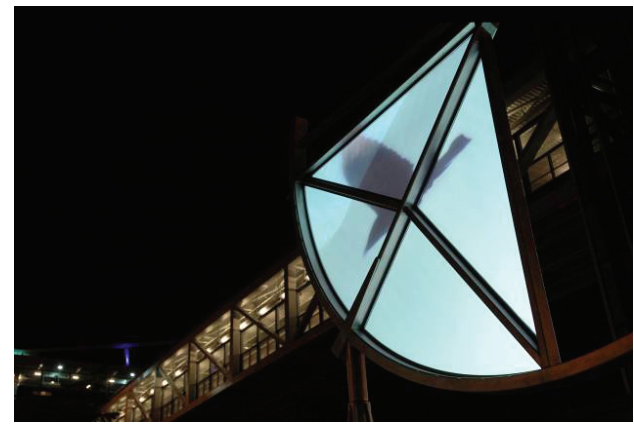

Figure 9: Celestial Navigations; Seattle's SeaTac Airport Plaza

Locating the Motion Studies footage within the context of a major International Airport brings a least a peripheral awareness of the science of flight to thousands of viewers a day. 

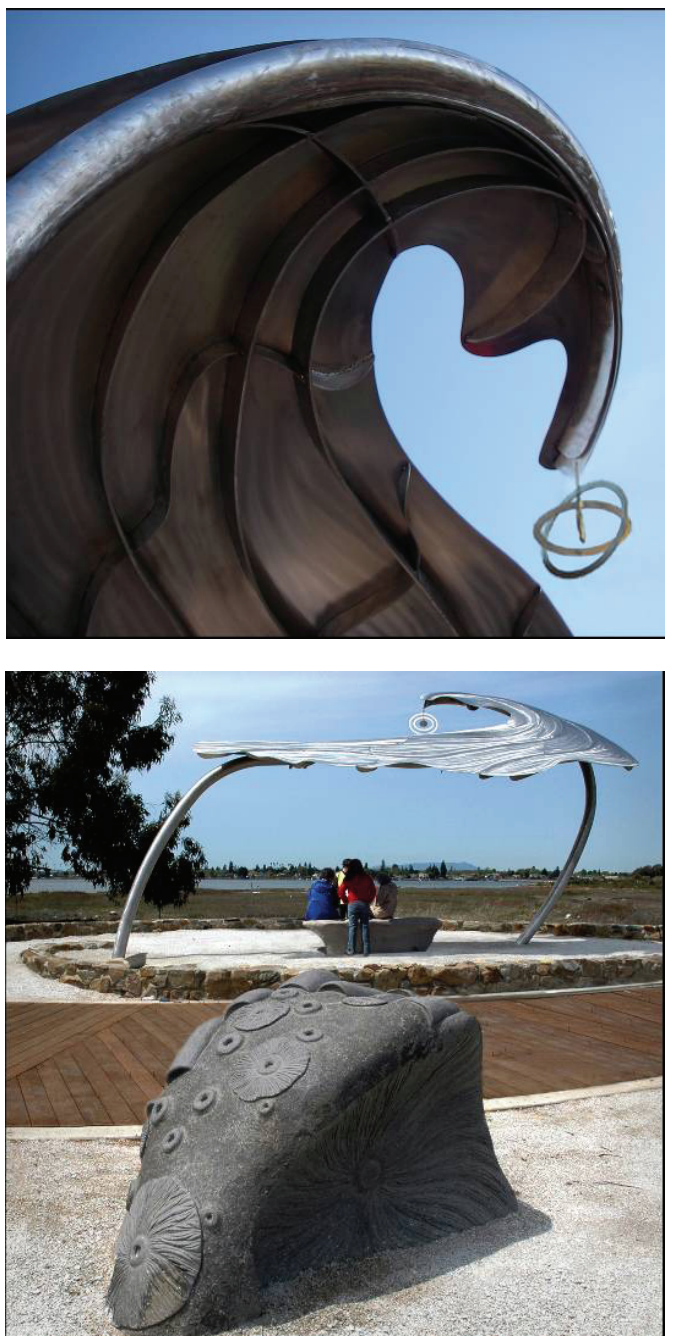

Figures 10 \& 11: Fluid Dynamics; Viewing shelter on San Francisco Bay. Both design and structural engineering are based on fluid dynamics (w Otani)

Other projects tied less to the collaboration nevertheless grew directly from it. These include a mile long migratory pollinator habitat 'Oasis' in Phoenix, Arizona, where D'Agostino incorporated the principals of Fluid Dynamics into landscape design for an interpretive landscape. Using Tobalske's research on the biomechanics of hummingbird flight, and research on the role of migratory pollinators being conducted at the Sonoran Desert Museum in Tucson, Arizona, .D'Agostino persuaded a sceptical Park Department to plant a mile long pollinator habitat corridor in place of previously specified decorative plantings. At a habitat restoration project at Smith and Bybee Lakes, in Portland Oregon, wing images from Dr Tobalske's flight studies were incorporated into carved cedar 'Habitat Trees' for migratory birds, and the forms of the engineered wetlands central to the restoration were based on the fluid dynamics of flight. 'Fluid Dynamics,' a wildlife viewing area on San Francisco Bay, is of particular interest. Sited on a critical node of the Pacific Migratory Bird Flyway, the entire site design is based on the principals of fluid dynamics, drawing on both the characteristic shapes of the flyway itself and on the form of the Pacific Current as it sweeps up the California Coast not far from the project site. The twenty six foot long stainless steel viewing shelter takes its overall form from the Pacific Current. Structural engineering for the shelter is also based on fluid dynamics and uses the characteristic wire frame grids of the LaVision software analysis of fluid motion as its primary structural design principal. Working with structural engineers, the team was able to achieve a structural design that reflects the natural dynamic of the bay side site. The shelter contains not a single right angle, as illustrated in figures 7 and 8 . The surrounding terraced landscape, also based on fluid dynamics, was reclaimed from an urban spoils dump and has been replanted with native species used as food sources by migrating birds. These projects were an outgrowth of insights D'Agostino gained through her work in the flight lab. At the beginning of the collaboration, $\mathrm{Dr}$ Tobalske said that he hoped that better understanding of their flight might ultimately help preserve the habitat of the birds he studies. In a small way, D'Agostino's work as both a gallery and public artist has been able to further this goal, by giving viewers an experience of moving through space along pathways and through landscapes more in tune with natural principals.

\section{NEXT STEPS; ART PROCESSES AND PRACTICE}

After the successful completion of Motion Studies, Dr. Tobalske arranged for D'Agostino to obtain a reduced cost copy of the DaVis and LaVision software used in the flight lab, from the company that developed it. This software is used not only for ornithological flight studies but for the analysis of airflow and fluid -dynamics in a host of other sciences, as well as many engineering fields. For example the Boeing Corporation in Seattle uses the identical software with a much larger wind tunnel to engineer its aircraft.

In the art world the software's ability to visualise the velocity and direction of motion in vivid colours, and to plot the flow of air, makes it an intriguing tool for the abstraction of any moving image. It has an infinitely expandable and variable palette, ranging from the vivid colours and grids of Motion Studies, to effects which take on the look of charcoal drawings or monoprints. D'Agostino intends to expand the range of subjects she edits using the LaVision software to include a contemporary dance collaboration. In a current project, she is again working with scientists for a public art commission 
at a local university. Many of these scientists also use computational fluid dynamics in their work and videos generated in their labs will become part of this project.

To expand the interactivity of this and other video installation projects, D'Agostino is investigating the potential integration of programming with Max MSP Jitter and other interactive technologies into her work and will be working with a programmer this year to study how interactive technology can be integrated into the Motion Studies series of art works.

\subsection{Next Steps; Scientific Research}

Inevitably, there are constraints associated with working with wild animals in a laboratory. Much of the recent research on flying birds involves the use of wind tunnels in which the animal flies within a chamber through which air is drawn using a powerful fan (Tobalske et al., 1997; Park et al., 2001; Spedding et al., 2003; Rosén et al., 2004). There may be effects of the wind tunnel upon flight performance due to the confined area in which the birds are flying, the peculiar aerodynamics due to being surrounded by walls (Rayner, 1994), as well as the stress upon the animal due to being in a noisy environment and being surrounded by humans. Almost no studies have explored the effects of the wind tunnel upon flight performance. Working with cinematographer Harry Dawson under controlled conditions in natural settings will allow us to compare flight behaviour in the wild with data from the controlled environment of the laboratory.

Generally, studies of wing and body motion in birds require manual digitisation of video or film images (Tobalske et al. 1997; Park et al. 2001; Rosen et al. 2004). This work is tedious, and it is limited in the extent to which multiple animals may be tracked simultaneously. For the present study, we propose to adapt the use of our gigital particle image velocimetry equipment to auto-track birds as they fly in the field. We have already successfully used this method to obtain preliminary (non-calibrated) data of swifts coming in to roost at the Portland, Oregon field site (D'Agostino et al. 2008). The LaVision DPIV system that we use will track any objects that may be distinguished via contrast, from sub-micron oil particles imaged using a laser in an interrogation area that is $20 \times 20 \mathrm{~cm}$ in size (Raffel et al., 2000; Spedding et al., 2003; Warrick et al., 2005; Henningsson et al., 2008), to birds illuminated by sunlight within a flock in an interrogation area of $100 \times 100 \mathrm{~m}$ (D'Agostino et al., 2008). To date, DPIV software has not been used to track bird velocity in the field. Our proposed research will provide several advances in our understanding of bird flight. Additionally, this research will advance the use of technology by demonstrating that software and video equipment presently used for detailed studies of particle movement in the wake of flying animals (Spedding et al. 2003; Warrick et al. 2005; Henningsson et al. 2008) may be adapted to auto-track small animals flying together in a flock. Ultimately, the ability to auto track animals in a flock will further our understanding of the ecological and evolutionary significance of flocking behaviour (Caraco et al. 1982; Lee et al. 2006).

\section{CONCLUSION}

Our collaborative work on the experimental video and installation Motion Studies, speaks strongly to the fertile cross pollination that is made possible by the digital revolution. In the course of our shared work intriguing new directions for investigation opened up for both the artists and the scientists involved in the project. The ability to convey scientific insights to the general public in a compelling way is one outgrowth of the collaboration. New means to see and attempt to express both the naturally invisible and the poetically ineffable, are perhaps the most exciting outgrowth of our shared work. We see Motion Studies as a beginning - stayed tuned.

\section{REFERENCES}

Caraco, T., Martindale, S. and Pulliam, H.R. (1982). Avian flocking in the presence of a predator. Nature 285, pp. 400-401.

D'Agostino, F., Tobalske, B.W. and Dawson, H. (2008). Motion studies: Elizabeth Leach Gallery, Portland, OR, 3-26 April 2008.

Henningsson, P. Spedding, G.R. and Hedenstrom, A. (2008). Vortex wake and flight kinematics of a swift in cruising flight in a wind tunnel. J. Exp. Biol. 211, pp. 717-730.

Lee, S.H, Pak, H.K., Chon, T.S. (2006). Dynamics of prey-flock escaping behavior in response to predator's attack. J. Theor. Biol. 240, pp. 250-259.

Park, K. J., Rosén, M. and Hedenström, A. (2001). Flight kinematics of the barn swallow Hirundo rustica over a wide range of speeds in a wind tunnel. J. Exp. Biol. 204, pp. 2741-2750.

Raffel, M., Willert, C. and Kompenhans, J. (2000). Particle Image Velocimetry: A Practical Guide. Berlin: Springer-Verlag.

Rayner, J. M. V. (1994). Aerodynamic corrections for the flight of birds and bats in wind tunnels. $J$. Zool., Lond. 234, pp. 537-563. 
Rosén, M. W., Spedding, G. R. and Hedenström, A. (2004). The relationship between wingbeat kinematics and vortex wake of a thrush nightingale. J. Exp. Biol. 207, pp. 4255-4268.

Spedding, G. R., Rosén, M. and Hedenström, A. (2003). A family of vortex wakes generated by a thrush nightingale in free flight in a wind tunnel over its entire natural range of flight speeds. J. Exp. Biol. 206, pp. 2313-2344.
Tobalske, B. W., Olson, N. E. and Dial, K. P. (1997). Flight style of the black-billed magpie: variation in wing kinematics, neuromuscular control, and muscle composition. J. Exp. Zool. 279, pp. 313-329.

Warrick, D. R., Tobalske, B. W. and Powers, D. P. (2005). Aerodynamics of the hovering hummingbird. Nature 435, pp. 1094-1097. 